\title{
Detection of the herpesviral hematopoietic necrosis disease agent (Cyprinid herpesvirus 2) in moribund and healthy goldfish: validation of a quantitative PCR diagnostic method
}

\author{
A. E. Goodwin*, G. E. Merry, J. Sadler \\ University of Arkansas at Pine Bluff, Aquaculture/Fisheries Center, 1200 N. University Dr., Mail Slot 4912, Pine Bluff, \\ Arkansas 71601, USA
}

\begin{abstract}
Cyprinid herpesvirus 2 (CyHV-2) is a pathogen of goldfish Carassius auratus auratus L. that causes herpesviral hematopoietic necrosis (HVHN) disease. The disease is associated with necrosis of hematopoietic tissues and anemia with high mortality. We have developed a real time 5'-nuclease PCR method (Taqman) that quantitatively detects CyHV-2 with a linear response over 8 logs of target concentration. The coefficient of variability on replicate samples tested on different days was $13 \%$ and the calculated sensitivity approached 1 target molecule per reaction. The assay does not cross-react with other similar fish herpesviruses, including CyHV-1 (carp pox) and CyHV-3 (koi herpesvirus), but reliably detects known CyHV-2 positive fish. The assay detects CyHV-2 not just in clinical cases of HVHN but also in apparently healthy 1 yr old goldfish fingerlings and even in 3 to 5 yr old broodfish.
\end{abstract}

KEY WORDS: Herpes · Virus · Goldfish · Quantitative PCR · CyHV-2

\section{INTRODUCTION}

Herpesviral hematopoietic necrosis (HVHN) is a disease of goldfish Carassius auratus auratus (Linnaeus, 1758) that has been reported in Japan, Australia, Taiwan and the USA. Mortality associated with HVHN can approach 100\% (Jung \& Miyazaki 1995, Groff et al. 1998, Chang et al. 1999, Stephens et al. 2004, Goodwin et al. in press). Herpesviral hematopoietic necrosis is caused by Cyprinid herpesvirus 2 (CyHV-2), a member of the cyprinid herpesvirus group that includes carp pox (CyHV-1) and koi herpesvirus (CyHV-3) (Waltzek et al. 2005). Reports of losses attributed to HVHN are limited to the 5 publications noted above, but the apparent rarity of HVHN may be due more to the lack of a sensitive and specific diagnostic test, rather than a reflection of the true incidence of the disease.
The diagnosis of HVHN has been based in most cases on histological findings and the demonstration, by electron microscopy, of herpesviral particles in tissues of infected fish (Jung \& Miyazaki 1995, Groff et al. 1998, Chang et al. 1999, Stephens et al. 2004). Cyprinid herpesvirus 2 has been cultured on cyprinid cell lines but the virus is lost after about 4 passages in vitro (Jung \& Miyazaki 1995). There is a single report of the successful development of a goldfish fin cell line that supports sequential passage of goldfish herpesvirus (Li \& Fukuda 2003), but distribution of the cell line is limited and propagation of the virus using this cell line in other laboratories has proven difficult (Gilad et al. 2004). More recently, several cases of HVHN in the USA were diagnosed and confirmed by degenerate PCR of the CyHV-2 DNA polymerase gene followed by DNA sequencing (Goodwin et al. 2006), but this method is time consuming and too technically 
demanding for most diagnostic work or for large research studies.

Little is known about the epidemiology of CyHV-2, but characteristics of other, better studied, fish herpesviruses indicate that $\mathrm{CyHV}-2$ will be difficult to control or eradicate. Koi herpesvirus (KHV or CyHV-3) spreads very easily and produces high mortality in wild and domestic stock of common carp and koi Cyprinus carpio (Hedrick et al. 2000). Koi herpesvirus is closely related to CyHV-2 and recent studies have shown that fish surviving acute disease carry the virus for at least several months post-infection (Gilad et al. 2004). These latently infected fish are anecdotally implicated in the spread of KHV to new populations. Channel catfish herpesvirus (CCV or IcHV-1) produces latent infections in channel catfish surviving acute infections (Gray et al. 1999, Stingley et al. 2003) and the virus may even be vertically transmitted (Hanson et al. 2004).

The detection of carrier status for herpesviruses is difficult because latently infected animals may carry the viral genome without producing infectious particles that could be detected in cell culture or by serological methods (Lamers et al. 1985, Cohrs \& Gilden 2001, Kamal et al. 2004). Another approach is to look for antiviral antibodies that would indicate prior virus exposure, but in fish the duration of detectable titers against specific pathogens is quite variable (Vestergaard \& Jørgensen 1982, Eggset et al. 1997, Bricknell et al. 1999). More problematic is that antibodies may not be present in fish that carry the virus but that have not yet developed the acute disease. Because of the limitations of culture and serological methods, the detection of latent herpesviral infections most often relies on the detection of the viral genome by PCR (Gray et al. 1999, Gilad et al. 2004).

Traditional PCR tests are useful for the detection of viral genomes in extracted fish or cell culture DNA, but these methods provide only a 'yes or no' answer that does not differentiate between a fish dying from an active viral infection and one that is dying of some other disease but carrying the virus in its latent form. The answer to this problem is the development of quantitative PCR methods that can be used to differentiate between the high viral loads typical of an active infection and the much lower loads that may be associated with latency or carrier status. Other advantages of quantitative PCR over conventional PCR are that it is faster, that methods using both primers and fluorescent probes are more specific, and that there is a smaller chance of false positives caused by the contamination of new reactions with the products of previous PCR assays (Mackay et al. 2002). In our work, we report the development and validation of a quantitative 5 '-nuclease PCR method (Taqman assay; Heid et al. 1996) to detect CyHV-2 and its successful use to detect viral DNA in both moribund and apparently healthy goldfish.

\section{MATERIALS AND METHODS}

Primers and probe. Primer and TaqMan probe sequences (Table 1) were selected from the CyHV-2 DNA polymerase gene sequence (Genbank AY939863, Waltzek et al. 2005) using Beacon Designer 4.0 (PREMIER Biosoft International). The probe was labeled at the $5^{\prime}$ end with the fluorescent reporter FAM and at the 3 ' end with the quencher BHQ-1 (Integrated DNA Technologies). The PCR product is $170 \mathrm{bp}$.

PCR conditions. Real-time PCR for CyHV-2 was performed in $12.5 \mu \mathrm{l}$ reactions containing $2.5 \mu \mathrm{l}$ of template, $0.5 \mu \mathrm{l}$ each of forward and reverse primers $(10 \mu \mathrm{M}$ each), $0.5 \mu \mathrm{l}$ of FAM-labeled probe $(10 \mu \mathrm{M})$, $6.25 \mu \mathrm{l}$ of $2 \mathrm{X}$ supermix (Bio-Rad), and $2.25 \mu \mathrm{l}$ of water. The PCR thermal profile consisted of an initial incubation of 2 min at $95^{\circ} \mathrm{C}$ followed by 35 cycles of $45 \mathrm{~s}$ at $58^{\circ} \mathrm{C}, 45 \mathrm{~s}$ at $72^{\circ} \mathrm{C}$, and $30 \mathrm{~s}$ at $95^{\circ} \mathrm{C}$, and a final extension of $2 \mathrm{~min}$ at $72^{\circ} \mathrm{C}$. The annealing temperature was selected in preliminary experiments where DNA from known positive fish was run in triplicate at 8 different annealing temperatures. The PCR was run on a real time PCR machine (iCycler; Bio-Rad) using the manufacturer's software (iQ Real-Time Detection System). The concentration and purity of all template DNA was verified using spectrophotometry (Nanodrop ND-1000).

Standard curve/dynamic range. To determine the dynamic range of the Taqman assay, the PCR product was purified using a GFX PCR DNA \& Gel Band Purification kit (Amersham Biosciences), then cloned and transfected into Escherichia coli (TOPO-TA cloning kit for sequencing, Invitrogen). Plasmid DNA was purified by an Amersham Biosciences GFX Microplasmid Prep kit. The concentration was determined by spectrophotometry, then serially diluted from $10^{8}$ down to 1 copy per reaction. The PCR efficiency and correlation coefficient were calculated from the standard curve (iQ Real-Time Detection System; Bio-Rad).

To examine the possibility that non-target DNA would interfere with the assay, additional standard curves were developed by serial dilution of plasmid DNA alone, and by serial dilution of plasmid DNA in the presence of

Table 1. Primer and probe sequences for the detection of Cyprinid herpes-virus $2(\mathrm{CyHV}-2)$ by quantitative Taqman PCR
Forward
5'-TCGGTTGGACTCGGTTTGTG-3'
Reverse 5'-CTCGGTCTTGATGCGTTTCTTG-3'
Probe $\quad 5^{\prime}$-FAM-CCGCTTCCAGTCTGGGCCACTACC-BHO1-3' 
CyHV-2 negative goldfish spleen DNA isolated according to manufacturer's instructions (Qiagen DNeasy tissue kit), using a $200 \mu \mathrm{l}$ elution volume. Serial 1:9 dilutions of plasmid were made from $2.63 \times 10^{8}$ to $2.63 \times$ $10^{1}$ copies per reaction. One series was diluted in TE buffer, the other in TE buffer containing enough spleen DNA such that each $12.5 \mu \mathrm{l}$ reaction received $600 \mathrm{ng}$. All were run in triplicate using the PCR protocol above. The PCR efficiency was calculated using the commercial software (iQ Real-Time Detection System).

To ensure that intact CyHV-2 DNA behaved the same as the plasmid, additional standard curves were developed using DNA extracted from the spleen and trunk kidney of a known CyHV-2 positive fish (Goodwin et al. 2006). The DNA was diluted with and without the same $600 \mathrm{ng}$ per reaction of spleen DNA described above. The PCR efficiency of dilution series of fish extracts with or without spleen DNA was compared to that of plasmid DNA alone.

Sensitivity of assay. Serial dilutions of the plasmid containing the CyHV-2 PCR product were amplified in quadruplicate by the protocol described above. Dilutions covered the entire dynamic range of the assay from $10^{8}$ copies per reaction down to 1 copy.

Precision. DNA from 3 known positive fish (Goodwin et al. 2006) was tested for CyHV-2 using the plasmid DNA standard curve. Each sample was run in triplicate 2 or 3 times on different days and the mean and standard deviation of the copy number was measured. A blank, using TE buffer in place of template, and negative fish tissue controls were included in triplicate for every run. The coefficient of variation (standard deviation/mean) was calculated for each sample.

Specificity of assay. To determine whether there was cross-reactivity (cross-amplification) with other herpes viruses, CyHV-1, CyHV-3 and IvHV-1 were subject to real-time PCR using our quantitative assay. The CyHV-1 DNA was obtained by extraction of DNA from typical carp pox lesions removed from the skin of koi. The CyHV-3 positive DNA was extracted from infected cell culture layers of KF-1 cells. The isolate was from a case occurring in California in 1996. The DNA was extracted when almost all cells in the culture showed the vacuolization typical of KHV. The IcHV-1 DNA was extracted from spleens of moribund channel catfish with active channel catfish virus disease confirmed by culture. The identities of the cyprinid control viral isolates were confirmed by PCR (Gilad et al. 2004, Waltzek et al. 2005)

To determine cross-reactivity in conventional PCR, the same thermal profile was followed, using $12.5 \mu \mathrm{l}$ reactions containing $2.5 \mu \mathrm{l}$ of template as described above, $0.5 \mu \mathrm{l}$ of each primer, $6.25 \mu \mathrm{l}$ of Sybergreen supermix (2X) (BioRad) and $2.75 \mu \mathrm{l}$ of water, but with no Taqman probe. Melting curves were determined in $0.5^{\circ} \mathrm{C}$ increments over 80 cycles. The PCR products were electrophoresed in agarose gels and stained with ethidium bromide.

Organ choice for CyHV-2 detection. Total DNA was extracted from the gill, liver, spleen, trunk kidney, gonad, swim bladder, and brain of 13 goldfish using the Qiagen kit and following the manufacturer's instructions for both fresh and formalin fixed tissues, then tested for CyHV-2 using the quantitative PCR assay described above using the plasmid for the standard curve. All fish tested were samples from 4 clinical cases of HVHN (Goodwin et al. in press) but not all fish tested had clinical signs of the disease. Due to the small size of some fish, and to the necessity to obtain samples for other diagnostic procedures, not all organs were tested from every fish. In one case, DNA was extracted from the gill, visceral organs, and brain of a moribund fish frozen by the fish owner, then thawed in $10 \%$ neutral buffered formalin and held for $1 \mathrm{yr}$ prior to DNA extraction.

CyHV-2 detection in fish without clinical signs of HVHN. Apparently healthy 3 to 5 yr old goldfish broodstock $(n=590)$ were obtained from 2 different goldfish breeders during March and April 2005. Trunk kidney and spleen tissue was removed from the broodfish, pooled in groups of 5 fish per pool, and then DNA for PCR was extracted using the Qiagen kit. Apparently healthy 7 to 8 mo old fingerling goldfish $(n=135)$ were obtained from 3 goldfish breeders, also during March and April 2005, and analyzed as 4 separate lots because they were collected on different days or from groups of fish raised in separation. The fish were submitted to the laboratory for a routine culture-based virological survey, so tissues were handled as if for cell culture. Trunk kidney and spleen tissues from all of the fingerlings were pooled at 5 fish per pool, homogenized in Hank's balanced salt solution, and centrifuged for $10 \mathrm{~min}$ at $10000 \times g_{\text {; }}$ then DNA for PCR was extracted from the cell pellet using the Qiagen kit. The CyHV-2 copy numbers in the extracted broodfish and fingerling DNA were determined by PCR as described above and read off the plasmid DNA standard curve. All samples were run in triplicate.

\section{RESULTS}

\section{Standard curve/dynamic range}

Standard curves run using purified plasmid DNA as the template were linear over 8 logs of template concentration. The correlation coefficient for the best straight line drawn through the threshold cycle values for each concentration was 0.98 and PCR efficiency was $100.0 \%$ (Fig. 1). 


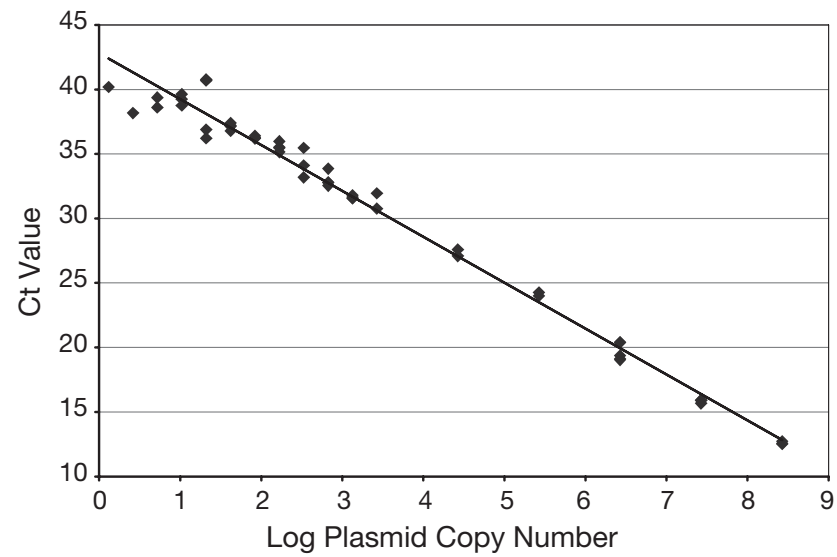

Fig. 1. Standard curve showing threshold cycle value (Ct) for Cyprinid herpesvirus 2 (CyHV-2) detection by Taqman PCR. The standard is a plasmid containing the Taqman PCR product. Correlation coefficient for the best straight line is 0.98

The PCR efficiencies for plasmid DNA and known positive fish DNA, both alone and spiked with $600 \mathrm{ng}$ spleen DNA per reaction, were similar. The plasmid alone in TE buffer had a correlation coefficient of 0.999 and PCR efficiency of $100.5 \%$ and CyHV-2 positive fish DNA diluted in TE had a correlation coefficient of 0.999 and PCR efficiency of $106.2 \%$. In the presence of 600 ng spleen DNA, the plasmid produced a correlation coefficient of 0.999 and efficiency of $99.9 \%$, while the CyHV-2 positive fish DNA had a correlation coefficient of 0.999 and PCR efficiency of $94.7 \%$.

\section{Sensitivity of assay}

Using quantitative PCR of serial dilutions of our plasmid, quadruplicate reactions calculated to have at
Table 2. Cyprinid herpesvirus 2 (CyHV-2) copy number by quantitative PCR. Data from 3 positive goldfish Carassius auratus auratus tested multiple times on different days. All numbers shown are in millions of copies per $\mu \mathrm{g}$ of host DNA. SD: standard deviation; CV: coefficient of variation; nd: not done

\begin{tabular}{|lcccccr|}
\hline Sample & Run 1 & Run 2 & Run 3 & Mean & SD & CV \\
\hline 1 & 190 & 140 & 200 & 177 & 32 & 18 \\
2 & 223 & 254 & 266 & 248 & 22 & 9 \\
3 & 164 & 139 & nd & 152 & 18 & 11 \\
\hline
\end{tabular}

least 20 copies of the plasmid tested positive for CyHV-2 every time (Fig. 1). Reactions calculated to contain 10 copies were positive 3 out of 4 times. Reactions with $5,2.5$, and 1 copy were positive in 2 of 4 , 1 of 4 , and 1 of 4 times respectively.

\section{Precision}

The PCR assays replicated on the same sample from fish with clinical CyHV-2 disease but on different days demonstrated low variability (Table 2). The coefficients of variation of replicate tests were 9 to $18 \%$ with an average of $13 \%$.

\section{Specificity of assay}

The quantitative Taqman assay produced no crossreaction with CyHV-1, CyHV-3, or IcHV-1. Using the same primers but no probe in a traditional PCR reaction produced a product with CyHV-1 and CyHV-2 as the template, but not with CyHV-3 or IcHV-1. Align-

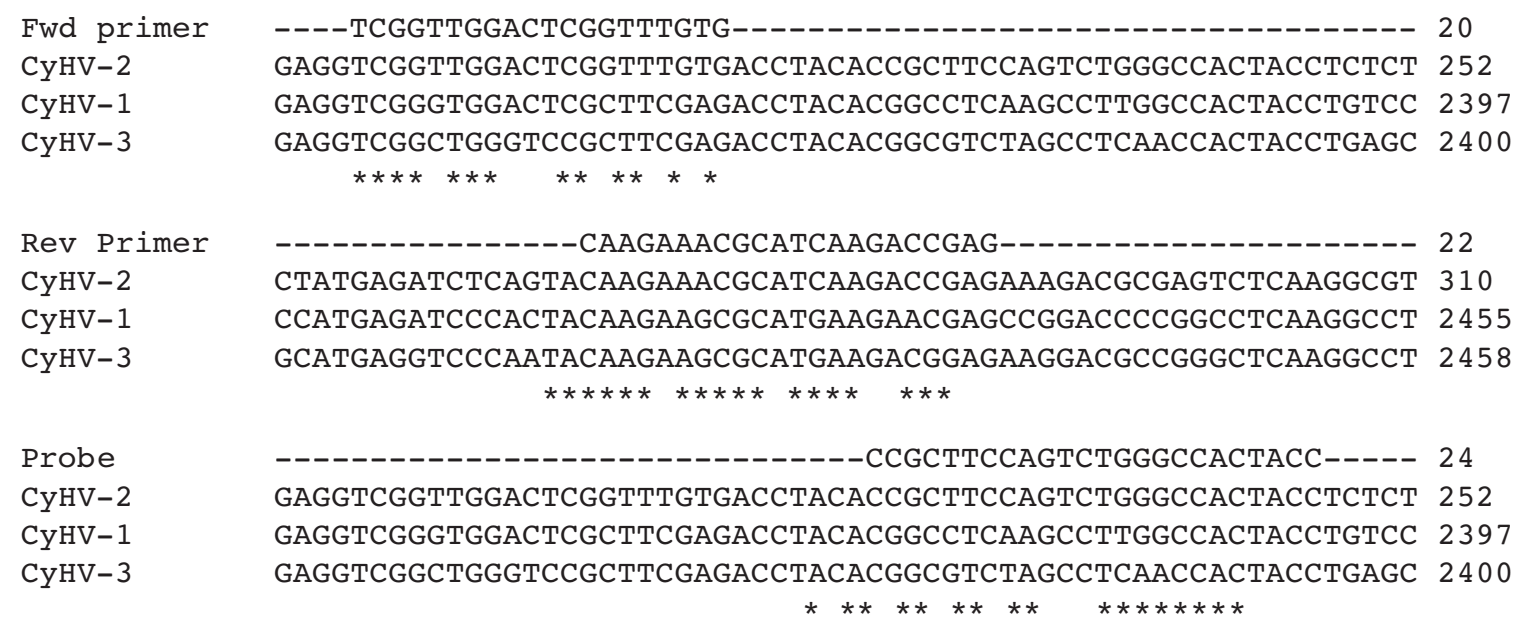

Fig. 2. Comparison of primer and probe sequences for TaqMan PCR of Cyprinid herpesvirus 2 (CyHV-2) to published sequences for other cyprinid herpesviruses (Waltzek et al. 2005). Sequences shown are from Genbank (CyHV-1, AY939868; CyHV-2, AY939863; CyHV-3, AY939862). Conserved nucleotides are noted with an asterisk 
Table 3. Comparison of Cyprinid herpesvirus 2 (CyHV-2) copy number measured by quantitative PCR of individual organs from goldfish Carassius auratus auratus sampled from populations with clinical signs of CyHV-2 infection. All numbers are in thousands of copies per $\mu \mathrm{g}$ DNA. Fish numbers denote clinical cases, lower case letters are individual fish; nt: not tested

\begin{tabular}{lccccccccc|}
\hline Sample & Gill & Liver & Spleen & $\begin{array}{c}\text { Trunk } \\
\text { kidney }\end{array}$ & Gonad & $\begin{array}{c}\text { Swim } \\
\text { bladder }\end{array}$ & Mixed \\
viscera
\end{tabular}

Table 4. Cyprinid herpesvirus 2 (CyHV-2) as thousands of copies per $\mu \mathrm{g}$ goldfish Carassius auratus auratus tissue DNA as detected by Taqman PCR. Samples were of pooled trunk kidney tissue from fish with no clinical signs of CyHV-2 infection

\begin{tabular}{|c|c|c|c|c|c|c|}
\hline \multirow[t]{2}{*}{ Fish } & \multirow{2}{*}{$\begin{array}{l}\text { Pools of } \\
5 \text { tested }\end{array}$} & \multirow{2}{*}{$\begin{array}{c}\text { Pools } \\
\text { positive }\end{array}$} & \multirow{2}{*}{$\begin{array}{l}\text { Mean of } \\
\text { positives }\end{array}$} & \multirow[t]{2}{*}{ SD } & \multicolumn{2}{|c|}{ Range } \\
\hline & & & & & Low & High \\
\hline Fingerling & 15 & 15 & 13000 & 33000 & 1.1 & 130000 \\
\hline Fingerling & 7 & 2 & 0.82 & 0.67 & 0.047 & 1.3 \\
\hline Fingerling & 5 & 0 & - & - & - & - \\
\hline Fingerling & 10 & 10 & 98000 & 110000 & 1.3 & 310000 \\
\hline Broodfish & 118 & 42 & 46 & 150 & 0.12 & 740 \\
\hline Total fish & 775 & & & & & \\
\hline
\end{tabular}

ment of the primer and probe sequences with CyHV-1, CyHV-3, and IcHV-1 sequences show that the primer sequences are most similar to CyHV-1 but that there are 4 mismatches in the forward primer, 3 in the reverse, and 5 in the Taqman probe (Fig. 2).

\section{Organ choice for CyHV-2 detection}

Of the organs tested, the spleen and trunk kidney consistently had the highest copy number of CyHV-2 DNA, but viral DNA was also often detected in the liver, gonad, swim bladder and brain (Table 3). In 1 fish (1a in Table 3 ) the spleen and trunk kidney tested neg- ative but viral DNA was detected in the gonad and brain. The fish in groups 1 and 3 had no detectable viral DNA in the gill, but gill tissues from groups 2 and 4 were strongly positive. While DNA from fixed tissue cannot be directly compared to that from fresh samples, CyHV-2 DNA was successfully detected in Fish 4 even though the tissue had been both frozen and formalin fixed.

\section{CyHV-2 detection in fish without clinical signs of $\mathrm{HVHN}$}

There was considerable variability in viral DNA load among apparently healthy goldfish tested by our PCR (Table 4). In groups of fingerlings the incidence of detectable CyHV-2 varied from all to none of the pools tested. In groups of both fingerlings and broodfish, low incidence (the percentage of pools positive) of detectable CyHV-2 was associated with low copy numbers (820 and $46000 \mathrm{\mu g}^{-1}$ host DNA). In the 2 groups of fingerlings where $100 \%$ of the pools tested positive, the mean copy number was at least 300 times higher (13000000 and $98000000 \mu^{-1}$ host DNA). In 3 to 4 yr old broodfish, 42 of 118 pools were positive for CyHV-2 and the average copy number in positive pools was $46000 \mu^{-1}$ host DNA. Numbers of copies and incidence for individual fish are not known because the number of positive fish per pool is unknown, but may have varied from 1 to 5 .

\section{DISCUSSION}

The quantitative PCR method that we describe is specific for $\mathrm{CyHV}-2$ and did not detect the closely related fish herpesviruses CyHV-1, CyHV-3, and IcHV-1. The assay is quantitative over an 8 log range of target copy numbers with an efficiency approaching $100 \%$ (Fig. 1). The efficiency of the reaction is the same for intact CyHV-2 as it is for the plasmid, and efficiency is not impaired by the addition of comparatively large amounts of non-target DNA. The assay closely attains the hypothetical sensitivity limit of 1 target molecule per reaction (considering the potential for mole- 
cules to be lost on tube or tip walls, and the obvious improbability that all 4 dilutions to 1 molecule per reaction would actually end up with 1 molecule per tube). Quantitative results were very precise with independent runs returning very similar values even on different days with a coefficient of variability of just $13 \%$ (Table 2). All known positive fish tested positive using the assay.

Our finding that spleen, trunk kidney, and brain appear to be the best organs for CyHV-2 PCR is consistent with both the biology of HVHN and with standard protocols for fish virology. Published histological descriptions of HVHN all describe necrosis of the hematopoietic tissues, so it is reasonable to expect the virus to be present in large amounts in spleen and trunk kidney tissues (Jung \& Miyazaki 1995, Groff et al. 1998, Chang et al. 1999, Stephens et al. 2004). Standard protocols for fish virology suggest that the best organs for virus screening are pools of spleen, trunk kidney, and brain tissues (USFWS and AFS-FHS 2004). Pools of these organs would have been positive in all of our cases, with the brain apparently only being required for 1 positive fish (1a in Table 3). The results from gill tissue testing (Table 3 ) are interesting in that 2 of 4 groups of positive fish were strongly gill positive while the other groups were negative. This is probably more a result of an intense general viremia in groups 2 and 4 (Table 3 ) than it is indicative of different disease mechanisms among the 4 cases. Virus detected in gill samples may also have resulted from adherence of waterborne virus to gill tissues. It would be interesting to do further studies of organ distribution over time following CyHV-2 infection, but in the present study we chose to limit our investigation to fish with naturally occurring HVHN.

In the present study, we have chosen to report some of our results as copy numbers per $\mu \mathrm{g}$ of total DNA template. Reporting the results as copy number per mg of tissue would be problematic because of differences in DNA extraction efficiency among different organ types, and due to the effects of sample size and quality. While we find our copies for $\mu \mathrm{g}$ units to be very useful in comparing levels of infection, others may wish to report values on a per host cell basis. This can be approximated by dividing the number of $\mu \mathrm{g}$ of DNA tested by the molecular weight of the goldfish genome, or by doing a parallel quantitative PCR assay for a goldfish gene (Gilad et al. 2004). We did not use a goldfish gene in parallel because running the additional assays almost doubles requirements for machine time, labor, and reagents. Attempts to reduce the time and expense by using a duplex reaction for CyHV-2 and the common carp glucokinase gene (Gilad et al. 2004) were unsuccessful because the high copy number of the glucokinase gene out-competed the CyHV-2 reaction, producing a loss of CyHV-2 sensitivity (data not shown).

Application of our assay to healthy and moribund fish served not only to validate the assay, but also to provide some very interesting insights into HVHN disease and the potential for CyHV-2 latency. Studies looking for latency in other fish viruses have proven very difficult and copy numbers of KHV (CyHV-3) drop to levels almost undetectable by quantitative PCR within $62 \mathrm{~d}$ post infection (Gilad et al. 2004). Detection of latency of IcHV-1 has not been reported quantitatively, but detection of the viral genome by regular PCR required very sensitive nested reactions (Gray et al. 1999). This supports a conclusion that both of those viruses are present only in very low copy numbers in fish without clinical disease. Surprisingly, we were able to easily detect CyHV-2 in asymptomatic fingerling goldfish and even in broodfish that were several years old (Table 4). While we have not formally demonstrated that these cases involve latent infections, the high prevalence of the virus in fish of all ages, including young of the year, makes it likely that in broodfish we are detecting CyHV-2 at several years post infection. This implies that the CyHV-2 virus is present in a higher number in asymptomatic fish than are the other fish herpesviruses.

Two of the groups of apparently healthy goldfish fingerlings tested for CyHV-2 in our assay produced surprisingly high copy numbers (Table 4). Both of these groups of fish were sampled during the spring when HVHN is most likely to occur and both were shipped overnight in boxes with oxygen. It may be that the shipping stress triggered the onset of acute HVHN disease in these fish, but that they were not yet showing clinical signs at necropsy. It is also possible that the disease was developing before the fish were sampled and shipped, but the breeders supplying the fish reported no HVHN-like losses in those fish that remained behind in ponds on their facilities.

The importance of CyHV-2 in aquaculture is still unclear but there is now enough information to speculate on similarities to other fish herpesviruses. KHV is highly pathogenic, producing high mortality in exposed fish of all ages. Exposures outside of the temperature optimum of the disease seem to often induce a carrier state that develops into acute disease when temperatures enter the permissive range (Hedrick et al. 2000). CCV is very different and appears to have a very high incidence in channel catfish populations but only rarely produces disease, which occurs only in young of the year fish during periods of hot weather, often combined with other stressors like handling (Plumb 1978, Gray et al. 1999). Given that CyHV-2 is very widespread and that diagnoses are rare, it is tempting to assume that HVHN is more like CCV dis- 
ease than KHV, but the prior lack of good testing methods for HVHN may have led to an underestimation of its importance. Some breeders do report that they have experienced significant fall mortalities in goldfish, and that anemia often appeared to be involved.

For diagnostic laboratories without access to real time PCR machines, we also tested our primer set for conventional PCR. Using this assay and electrophoresis to detect products in ethidium bromide stained agarose gels, we have shown that the assay does crossreact with CyHV-1 but not with CyHV-3 or IcHV-1. This implies that much of the specificity of the assay is attributable to the Taqman probe (where 5 mismatches occurred between the probe sequence and that of CyHV-1; Fig. 2) and not to the primers. The occurrence of this cross-reaction with CyHV-1 is not ideal, but CyHV-1 is a koi and common carp pathogen that has not been detected in goldfish. Thus, while a standard PCR assay needs to be validated for diagnostic laboratories without access to quantitative PCR equipment, our primers may be useful for the interim in standard PCR for the diagnosis of acute disease in goldfish.

Our quantitative PCR method will facilitate future studies on both the impact of CyHV-2 and on its control and eradication. If breeders are truly experiencing significant mortality from HVHN, then farm-level eradication efforts may be warranted. However, the success of those efforts cannot be guaranteed until it is known if CyHV-2 is vertically transmitted and if that transmission is inside or on the outside of the gametes. We are currently working to answer this question.

\section{LITERATURE CITED}

Bricknell IR, King JA, Bowden TJ, Ellis AE (1999) Duration of protective antibodies, and the correlation with protection in Atlantic salmon (Salmo salar L.), following vaccination with an Aeromonas salmonicida vaccine containing ironregulated outer membrane proteins and secretory polysaccharide. Fish Shellfish Immunol 9:139-151

Chang PH, Lee SH, Chiang HC, Jong MH (1999) Epizootic of herpes-like virus infection in goldfish, Carassius auratus in Taiwan. Fish Pathol 34:209-210

Cohrs RJ, Gilden DH (2001) Human herpesvirus latency. Brain Pathol 11:465-74

Eggset G, Mikkelsen H, Killie JEA (1997) Immunocompetence and duration of immunity against Vibrio salmonicida and Aeromonas salmonicida after vaccination of Atlantic salmon (Salmo salar L.) at low and high temperatures. Fish Shellfish Immunol 7:247-260

Gilad O, Yun S, Zagmutt-Vergara FJ, Leutenegger CM, Bercovier H, Hedrick RP (2004) Concentrations of a koi herpesvirus (KHV) in tissues of experimentally infected Cyprinus carpio koi as assessed by real-time TaqMan

Editorial responsibility: Jo-Ann Leong,

Kaneohe, Hawaii, USA
PCR. Dis Aquat Org 60:179-187

Goodwin AE, Khoo L, LaPatra SE, Bonar C, Key DW, Garner M, Hanson L (2006) Goldfish hematopoietic necrosis herpesvirus (Cyprinid herpesvirus 2) in the USA: molecular confirmation of isolates from diseased fish. J Aquat Anim Health 18:11-18

Gray WL, Williams RJ, Griffin BR (1999) Detection of channel catfish virus DNA in acutely infected channel catfish, Ictalurus punctatus (Rafinesque), using the polymerase chain reaction. J Fish Dis 22:111-116

Groff JM, LaPatra SE, Munn RJ, Zinkl JG (1998) A viral epizootic in cultured populations of juvenile goldfish due to a putative herpesvirus etiology. J Vet Diag Invest 10: 375-378

Hanson LA, Rudis MR, Petrie-Hanson L (2004) Susceptibility of channel catfish fry to Channel Catfish Virus (CCV) challenge increases with age. Dis Aquat Org 62:27-34

Hedrick RP, Gilad O, Yun S, Spangenberg JV and 5 others (2000) A herpesvirus associate with mass mortality of juvenile and adult koi, a strain of a common carp. J Aquat Anim Health 12:44-57

Heid CA, Stevens J, Livak KJ, Williams PM (1996) Real time quantitative PCR. Genome Res 10:986-994

Jung SJ, Miyazaki T (1995) Herpesviral haematopoietic necrosis of goldfish, Carassius auratus (L.). J Fish Dis 18: 211-220

Kamal MK, Lepisto AJ, Hendricks RL (2004) Immunity to latent viral infection: many skirmishes but few fatalities. Trends Immunol 25:5230-5234

Lamers CHJ, de Haas MJH, van Muiswinkel WB (1985) The reaction of the immune system of fish to vaccination: development of immunological memory in carp, Cyprinus carpio L., following direct immersion in Aeromonas hydrophila bacterin. J Fish Dis 8:253-262

Li X, Fukuda H (2003) In vitro culture of goldfish cell sensitive to goldfish herpesvirus. J Shanghai Fisheries Univ (Shanghai Shuichan Daxue Xuebao) 12:12-18

Mackay IM, Arden KE, Nitsche A (2002) Real-time PCR in virology. Nucleic Acids Res 30:1292-1305

Plumb JA (1978) Epizootiology of channel catfish virus disease. Mar Fish Rev 40:26-29

Stephens FJ, Raidal SR, Jones B (2004) Haematopoietic necrosis in a goldfish (Carassius auratus) associated with an agent morphologically similar to herpesvirus. Aust Vet J 82:167-169

Stingley RL, Griffin BR, Gray WL (2003) Channel catfish virus gene expression in experimentally infected channel catfish, Ictalurus punctatus (Rafinesque). J Fish Dis 26:487-493

USFWS and AFS-FHS (U.S. Fish and Wildlife Service and American Fisheries Society - Fish Health Section) (2004) Standard procedures for aquatic animal health inspections. In: AFS-FHS. FHS blue book: suggested procedures for the detection and identification of certain finfish and shellfish pathogens, 2004 edn. AFS-FHS, Bethesda, MD (available as CD)

Vestergaard PE, Jørgensen RD (1982) Egtved virus: temperature-dependent immune response of trout to infection with low-virulence virus. J Fish Dis 5:47-55

Waltzek TB, Kelley G, Stone DM, Way K, Hanso L, Fukuda H, Davison AJ, Hedrick RP (2005) Koi herpesvirus represents a third cyprinid herpesvirus (CyHV-3) in the family Herpesviridae. J Gen Virol 86:1659-1667

Submitted: July 12, 2005; Accepted: December 9, 2005

Proofs received from author(s): March 15, 2006 